\title{
Riesgos ocupacionales en estudiantes de veterinaria en Argentina
}

\author{
Tarabla, H.D. ${ }^{1}$; Molineri, A.I. ${ }^{2,3}$; Robin, H. ${ }^{4}$; Signorini, M.L. ${ }^{1,2}$ \\ ${ }^{1}$ Fac. Cs. Veterinarias, Univ.Nac.Litoral, Esperanza, Santa Fe, Argentina. \\ ${ }^{2}$ CONICET, INTA Rafaela, ${ }^{3} \mathrm{UNRaf},{ }^{4}$ Consejo Interuniv.Nacional. \\ E-mail: htarabla@fcv.unl.edu.ar
}

\begin{abstract}
Resumen
Tarabla, H.D.; Molineri, A.I.; Robin, H.; Signorini, M.L.: Riesgos ocupacionales en estudiantes de veterinaria en Argentina. Rev. Vet. 30: 1, 63-67, 2019. La práctica veterinaria expone a los individuos a una gran variedad de accidentes ocupacionales (AO) y enfermedades profesionales. Los objetivos de este trabajo fueron estimar: a) la frecuencia de $\mathrm{AO}$ y zoonosis, b) la percepción de los riesgos ocupacionales (PR), c) el uso de elementos de protección personal (EPP) y d) las asociaciones con potenciales factores de riesgo en estudiantes de veterinaria de Argentina. Se efectuó un estudio observacional transversal en $n=$ 778 estudiantes de 13 carreras de Veterinaria ubicadas en nueve provincias argentinas. Los datos fueron recolectados mediante cuestionario estructurado auto-administrado. El análisis estadístico incluyó $\chi^{2}, t$ de Student y correlaciones de Pearson y de Spearman. Dos tercios $(67,5 \%)$ había sufrido $\mathrm{AO}$ en la práctica con grandes y pequeños animales, con gran variabilidad entre universidades $(\mathrm{p}=0,002)$. Los AO más frecuentes estuvieron vinculados con la exposición solar excesiva, los animales y los elementos punzo-cortantes. Aunque la atención clínica fue percibida como de riesgo medio o bajo, los AO se produjeron mayoritariamente en dichas instancias. Las lesiones (punzaduras, cortes, rasguños y mordeduras) estuvieron localizadas principalmente en extremidades superiores. El 6\% de los estudiantes había sido diagnosticado con alguna zoonosis, siendo la dermatofitosis la más frecuente $(2,8 \%)$. A mayor antigüedad como estudiante de veterinaria, mayores fueron las PR $(p<0,005)$ y la frecuencia de $\mathrm{AO}(\mathrm{p}<0,034)$. Los guantes fueron los EPP más utilizados. En algunas prácticas, su uso fue más frecuente en las mujeres $(\mathrm{p}<0,03)$. No hubo asociaciones entre la frecuencia de uso de EPP y las PR o los años como estudiantes de veterinaria. Se concluye que los contenidos sobre seguridad ocupacional deberían ser ofrecidos desde los primeros años de la carrera. Esta práctica, unida al esfuerzo de los docentes para que los alumnos observen el cumplimiento de prácticas seguras podrían modificar las actitudes, reduciendo los riesgos de $\mathrm{AO}$ y zoonosis entre los estudiantes.
\end{abstract}

Palabras clave: estudiantes, veterinaria, salud ocupacional, exposición, riesgos.

\begin{abstract}
Tarabla, H.D.; Molineri, A.I.; Robin, H.; Signorini, M.L.: Occupational risks in veterinary students in Argentina. Rev. Vet. 30: 1, 63-67, 2019. Veterinary practice exposes individuals to a wide variety of occupational accidents $(\mathrm{OA})$ and occupational diseases. The objectives of this work were to estimate: a) the frequency of OA and zoonoses, b) the perception of occupational risks (RP), c) the use of personal protective equipment (PPE) and d) the association with potential risk factors in veterinary students of Argentina. A cross-sectional observational study was carried out in $\mathrm{n}=778$ students of 13 veterinary careers located in nine Argentine provinces. The data were collected through a structured self-administered questionnaire. Statistical analysis included $\chi^{2}$, Student's $t$ test and Pearson's and Spearman's correlations. Two thirds (67.5\%) had suffered OA in large and small animal practice, with great variability among universities $(\mathrm{p}=0.002)$. The most frequent accidents were linked to excessive sun exposure, animals and sharp instruments. Although clinical examinations were perceived as medium or low risk activities, OA occurred mostly in these instances. Lesions were located mostly in the upper limbs. Six percent of students had been diagnosed with a zoonosis, being dermatophytosis $(2.8 \%)$ the most frequent. The higher the number of years as a veterinary student, the higher the RP $(p<0.005)$ and the frequency of $O A(p<0.034)$. Gloves were the most used PPE. In some practices, women used PPE more frequently than men $(p<0.03)$. There were no associations between the frequency of use of PPE and RP or number of years as a veterinary student. It is concluded that occupational safety practices
\end{abstract}


should be offered from the first years of the career. This practice, along with teachers' efforts to enforce students' observation of safety practices could modify attitudes, reducing the risks of $\mathrm{OA}$ and zoonoses among students.

Key words: students, veterinary, occupational health, exposure, risks.

\section{INTRODUCCIÓN}

Los estudiantes de veterinaria se exponen tempranamente a peligros biológicos, físicos, ergonómicos y radiaciones. Los ingresantes a la carrera tienen, en general, bajos niveles de conocimientos sobre zoonosis y los alumnos cursantes pueden tener hábitos y comportamientos inapropiados para su prevención ${ }^{24,29}$. La carrera tiene una carga creciente de lecciones prácticas y la última fase consiste en guardias y trabajos hospitalarios donde las actividades médicas son efectuadas por los estudiantes.

Los accidentes, los brotes de enfermedades y las infecciones zoonóticas son frecuentes en los hospitales-escuela ${ }^{6,26}$, donde las instalaciones pueden tener deficiencias en bioseguridad y las superficies de contacto pueden estar contaminadas con patógenos zoonóticos $5,20,45$.

Por otra parte, las actividades realizadas al aire libre y el incremento de los contactos con los pacientes en las etapas avanzadas de la carrera podrían aumentar el riesgo de accidentes ocupacionales $(\mathrm{AO})$ y enfermedades profesionales.

Con respecto a los $\mathrm{AO}$, los datos publicados en revistas indexadas son escasos e involucran la frecuencia de accidentes con elementos punzo-cortantes ${ }^{34,40}$ y equinos ${ }^{33}$. Muy diferente es la situación relativa a las zoonosis. En una extensiva revisión bibliográfica sobre estas enfermedades en estudiantes de veterinaria efectuada recientemente, un $45 \%$ de los artículos describían casos individuales o brotes y el restante 55\% resultados de relevamientos serológicos.

Los casos fueron causados por bacterias (63\%), parásitos (19\%), virus (15\%) y hongos (3\%). Aunque las referencias incluían 24 países en los últimos 55 años, el 66\% provenía de países de Europa y América del Norte ${ }^{36}$. Por otra parte, las publicaciones sobre la frecuencia de enfermedades alérgicas también han sido originadas en países europeos ${ }^{22,35}$. En el resto del continente americano, las referencias sobre zoonosis en estudiantes se limitan a relevamientos serológicos de anticuerpos contra brucelosis, leptospirosis, toxoplasmosis y tripanosomiasis $7-9,19,21,24,27,31,32,41,42$.

Dado el alto nivel de enfermedades profesionales y AO registrados en la práctica veterinaria en general y en nuestro país en particular ${ }^{2,18}$, se planteó este trabajo con el objetivo de estimar la frecuencia de AO y zoonosis, la percepción de riesgos (PR), y el uso de elementos de protección personal (EPP) en estudiantes de veterinaria de Argentina, así como las asociaciones con potenciales factores de riesgo.

\section{MATERIAL Y MÉTODOS}

Se efectuó un estudio observacional transversal en $\mathrm{n}=778$ estudiantes avanzados de 13 carreras de veterinaria dependientes de universidades estatales y privadas, ubicadas en nueve provincias argentinas.

El tamaño de la muestra se correspondió con una frecuencia esperada del $50 \%$, un nivel de confianza del 99\% y un error absoluto del 3,5\%. Los datos fueron recolectados mediante cuestionarios estructurados y autoadministrados, previamente a la iniciación de jornadas de capacitación sobre riesgos laborales en veterinaria.

En todos los casos, las instituciones sedes de las jornadas determinaron las cohortes de estudiantes que tenían obligatoriedad de asistencia. Previo a la distribución del cuestionario, se explicó la finalidad e importancia de la encuesta, poniéndose énfasis en que el interés de las mismas no estaba en las actividades o actitudes de ningún estudiante en particular, sino en las frecuencias a nivel poblacional, por lo cual las respuestas debían ser anónimas.

Se tomaron cuatro características de los encuestados para relacionarlas con la ocurrencia de zoonosis o $\mathrm{AO}$, la percepción de los riesgos y el uso de elementos de protección (sexo, edad, antigüedad como estudiante de veterinaria y facultad donde cursaba). "Percepción del riesgo" fue definida como "la noción de la probabilidad que una actividad pueda causar daño en la salud o integridad física" del encuestado (OMS, 2002).

Estas percepciones y la frecuencia de uso de EPP fueron estimadas mediante escalas ordinales. El análisis incluyó $\chi^{2}, t$ de Student y correlaciones de Pearson y de Spearman.

\section{RESULTADOS}

El $52,8 \%$ de los entrevistados eran mujeres. En promedio, los estudiantes tenían $23,9 \pm 3,2$ años de edad y habían permanecido $6,0 \pm 2,5$ años en la carrera de veterinaria. Ambas variables estuvieron altamente correlacionadas $(r=0,77, p<0,001)$, por lo cual sólo se incluyó la mencionada en segundo término en la búsqueda de asociaciones con otras variables.

No hubo diferencias entre sexos en la cantidad de años transcurridos como estudiantes de la carrera. El $67,5 \%$ había sufrido AO, con gran variabilidad entre universidades $(p=0,002)$. Los accidentes más frecuentes estuvieron vinculados con quemaduras y golpes de calor por exposición solar excesiva, rasguños y mordeduras de los animales, así como heridas con elementos punzo-cortantes (Figura 1). 
Tabla 1. Frecuencia de estudiantes que sufrieron accidentes ocupacionales de acuerdo a la actividad y al animal involucrado ( $\mathrm{n}=778$ estudiantes).

\begin{tabular}{lcccccc}
\hline \multirow{2}{*}{ actividad } & \multicolumn{5}{c}{ animal } & \multirow{2}{*}{ total } \\
\cline { 2 - 6 } & bovino & equino & canino & felino & otros & \\
\hline cirugía & 4,9 & 3,0 & 11,6 & 4,2 & 0,3 & 24,0 \\
necropsia & 5,7 & 1,90 & 3,2 & 0,5 & 1,4 & 12,7 \\
rev.clínica & 9,8 & 7,7 & 10,3 & 8,1 & 2,8 & 38,7 \\
vacunación & 12,4 & 1,8 & 7,8 & 4,6 & 0,4 & 27,0 \\
tratamiento & 5,0 & 3,7 & 6,2 & 3,1 & 1,3 & 19,3 \\
otras & 7,7 & 3,2 & 2,2 & 1,0 & 1,9 & 16,0 \\
\hline total & 45,5 & 21,3 & 41,3 & 21,5 & 6,1 & $*$ \\
\hline
\end{tabular}

*La frecuencia supera el $100 \%$ dado que hubo estudiantes que sufrieron dos o más accidentes con más de una especie.

Quienes habían sufrido AO habían permanecido más años como estudiantes $(\mathrm{p}=0,034)$. Los accidentes se produjeron mayoritariamente al trabajar con bovinos y caninos. Aproximadamente cuatro de cada seis estudiantes sufrieron AO durante la revisión clínica de los pacientes (Tabla 1).

Los varones sufrieron atropellamientos por animales con mayor frecuencia que las mujeres $(p=0,005)$, mientras que ellas padecieron más rasguños, pinchazos, contusiones y contracturas $(\mathrm{p}<0,05)$. Las lesiones estuvieron localizadas mayoritariamente en extremidades superiores (Figura 2).

El 6\% de los estudiantes había sido diagnosticado como positivo a alguna zoonosis (dermatofitosis $2,8 \%$, brucelosis $1,2 \%$, toxoplasmosis $1,2 \%$, sarna sarcóptica $0,9 \%$, carbunco $0,1 \%$ ). Uno de los encuestados comunicó haber padecido dos enfermedades (dermatofitosis y brucelosis). No hubo asociaciones entre estos diagnósticos y la universidad, el sexo o la antigüedad como estudiante de veterinaria.

La frecuencia de estudiantes que percibieron una actividad de riesgo alto fue mayor en la manipulación de abortos y en las necropsias, especialmente en las especies bovina y equina (Tabla 2). Aunque las correlaciones entre las percepciones en las distintas actividades fueron significativas, los coeficientes fueron relativamente bajos.

Los valores más altos se encontraron en la manipulación de abortos y la atención de partos $(\mathrm{r}=0,41)$, necropsias $(\mathrm{r}=0,35)$ y vacunación antibrucélica $(\mathrm{r}=0,34)$. A mayor antigüedad como estudiante de veterinaria, mayores fueron las percepciones de los riesgos ocupacionales $(\mathrm{p}=0,005)$.

Las atenciones clínicas fueron percibidas mayoritariamente como de riesgo medio o bajo (Figura 3). Estas percepciones, así como la ati-
Tabla 2. Percepción de los riesgos ocupacionales en estudiantes $(\mathrm{n}=778)$.

\begin{tabular}{llccc}
\hline \multirow{2}{*}{ especies y actividades evaluadas } & \multicolumn{3}{c}{ percepción del riesgo (\%) } \\
\cline { 2 - 4 } & Bovina & 23,6 & 63,8 & 12,6 \\
& Equina & 30,7 & 56 & 13,3 \\
\multirow{4}{*}{ especie } & 10,5 & 65,5 & 24 \\
& Porcina & 6,7 & 55 & 38,3 \\
& Ovina & 9,0 & 60,3 & 30,7 \\
& pequeñas especies & 6,2 & 36,4 & 57,4 \\
\hline \multirow{4}{*}{ actividad } & aves de corral & 38,2 & 50,9 & 10,9 \\
& tacto rectal & 50,8 & 37,8 & 11,4 \\
& atención de parto & 32,7 & 47,9 & 19,4 \\
& Cirugía & 69,0 & 22,2 & 8,8 \\
& manipulación de aborto & 76,0 & 18,9 & 5,1 \\
& vacunación antibrucélica & 54,2 & 32,2 & 12,6 \\
& podología bovina & 20,8 & 45,6 & 27,6 \\
\hline
\end{tabular}

nente a la vacunación antibrucélica, fueron mayores en mujeres que en hombres $(p<0,01)$.

El uso de EPP "siempre" varió entre actividades, siendo los guantes los elementos más utilizados (Figura 4). Las mujeres usaron "siempre" ropa específica en cirugías y partos, así como guantes en cirugías, con mayor frecuencia que los hombres $(\mathrm{p}<0,03)$.

No hubo asociaciones entre la frecuencia de uso de EPP y las percepciones de los riesgos o los años como estudiantes de veterinaria.

\section{DISCUSIÓN}

Más de dos tercios de los entrevistados había tenido algún $\mathrm{AO}$ y, como consecuencia, más de la mitad había sufrido lesiones. Al igual que en profesionales de grandes especies, la exposición solar excesiva fue muy frecuente entre los estudiantes relevados.

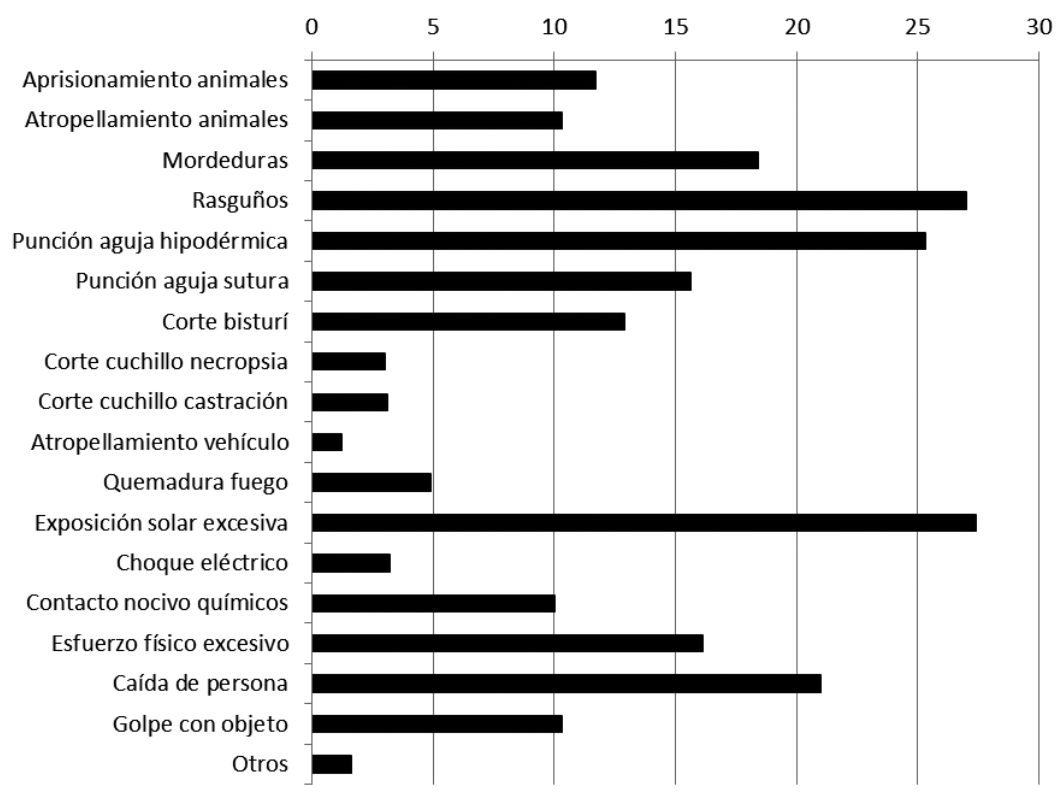

Figura 1. Frecuencia de estudiantes que sufrieron accidentes ocupacionales y características de los accidentes ( $\mathrm{n}=778$ estudiantes). 
Estos eventos causaron episodios agudos, pero la exposición crónica a rayos ultravioletas (UV) en veterinarios rurales es un evento aún no abordado. El trabajo al aire libre expone a niveles de UV más altos que los límites recomendados, aumentando el riesgo de cáncer de piel $^{4,37}$.

Esta probabilidad es tan alta, que algunos autores consideran que hay suficiente evidencia para considerarla una enfermedad ocupacional ${ }^{12}$. Si bien en este trabajo la exposición a rayos UV estuvo asociada a eventos agudos, la alta frecuencia de estudiantes afectados indica ausencia de prácticas seguras.

Aunque quienes trabajan al aire libre consideran que la protección es una responsabilidad de cada individuo ${ }^{38}$, en general, no adoptan medidas preventivas ${ }^{16,17}$. Coincidentemente a lo hallado en la joven población encuestada, la ausencia de cuidados ante las condiciones climáticas en veterinarios de grandes animales es más frecuente entre los profesionales de menor edad.

Un segundo aspecto de importancia fueron las heridas por elementos punzo-cortantes. Las agujas, los bisturís y los cuchillos son peligros que frecuentemente ocasionan $\mathrm{AO}$ entre los estudiantes ${ }^{40}$. Las heridas son causadas principalmente por agujas hipodérmicas y se localizan mayoritariamente en las extremidades superiores $^{2,10,25}$.

Los estudiantes tienen la tendencia de volver a tapar la aguja usada ${ }^{40}$ utilizando ambas manos ${ }^{34}$. Esta práctica, junto a la carencia de recipientes para su disposición segura, incrementan la probabilidad de heridas ${ }^{14,46}$.

Los $\mathrm{AO}$ se produjeron mayoritariamente durante las revisiones clínicas en bovinos y caninos. Paradójicamente, estas actividades son percibidas como de riesgo medio o bajo. Las PR más elevadas se observaron en equi$\operatorname{nos}(30,7 \%)$ y bovinos (23,7\%), cifras que descendieron a alrededor del $10 \%$ en pequeñas especies y porcinos.

Las mordeduras y rasguños, sin embargo, estuvieron entre los AO más frecuentes y los caninos entre las especies más involucradas. Estas frecuencias pueden estar relacionadas a las especies animales a las que se ven expuestos los estudiantes en las prácticas hospitalarias.

Aunque algunos pacientes conllevan un mayor riesgo por su agresividad, peso o masa corporal, la frecuencia de $\mathrm{AO}$ en profesionales argentinos es muy importante tanto en grandes como en pequeñas especies ${ }^{18}$. El manejo brusco por parte de los estudiantes y el dolor en los pacientes, han sido identificados como factores de riesgo de mordeduras de perros ${ }^{43}$.

Por otra parte, la mayor permanencia en la carrera, correlaciona con la mayor frecuencia de $\mathrm{AO}$, indicando una relación con el período de exposición. Sin embargo, esto no aumentó el uso de EPP, reafirmando que la experiencia no es un factor de protección ${ }^{43}$ y que las precauciones se suelen tomar a posteriori de la ocurrencia del accidente o enfermedad ${ }^{44}$.

Aunque el riesgo de lesiones aumenta con la exposición acumulada, los veterinarios con mayor antigüedad laboral usan EPP en actividades médicas con menor frecuencia que los más jóvenes. Los estudios observacionales transversales no permiten realizar inferencias sobre causalidad, por lo que su mayor utilidad no es la de proveer respuestas sino la de generar nuevas hipótesis.

La posibilidad que las conclusiones sean sesgadas por la presencia de factores de confusión o interacción, es siempre un evento latente. A modo de ejemplo, la frecuencia de brucelosis estuvo asociada al sexo y la edad en veterinarios rurales de la Provincia de Santa $\mathrm{Fe}^{31}$, pero en un análisis multivariado se demostró que en realidad, las mujeres eran más jóvenes que los hombres y que la asociación se explicaba simplemente por un mayor período de exposición de estos últimos.

En el presente estudio, quienes habían sufrido AO habían permanecido más años como estudiantes, existiendo diferencias en el tipo de accidente entre sexos. La posibilidad de que alguna de ellas haya actuado como factor de confusión fue descartada ante la inexistencia de asociación estadística entre permanencia en la carrera y sexo.

La gran mayoría de los veterinarios no considera que su propia seguridad esté en riesgo en sus prácticas diarias, lo cual puede representar una amenaza en términos de salud pública ${ }^{30}$. Por su parte, los estudiantes 


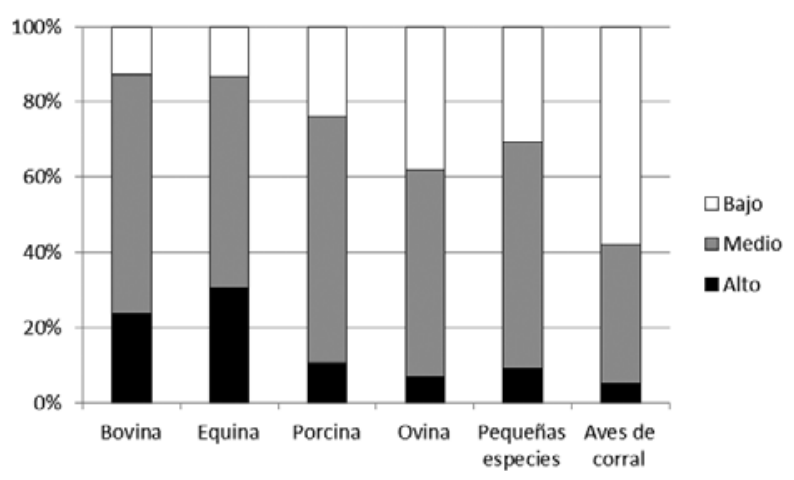

Figura 3. Percepción de los riesgos ocupacionales en las revisiones clínicas de acuerdo a la especie animal $(n=778)$.

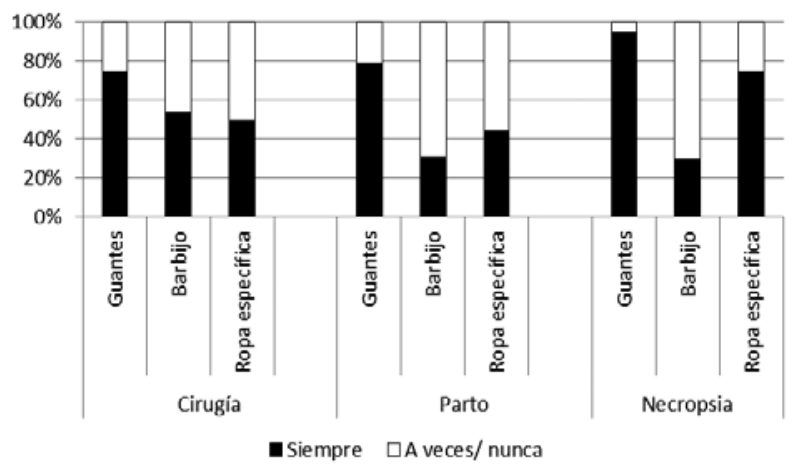

Figura 4. Frecuencia de estudiantes que usaron elementos de protección personal de acuerdo a la actividad realizada $(n=778)$.

pueden tener conocimientos, hábitos y comportamientos personales inadecuados para la prevención de $\mathrm{AO} y$ zoonosis $^{24}$.

Algunas prácticas seguras como la vacunación pre-exposición contra rabia por ejemplo, pueden tener poca aceptación ${ }^{29}$, resultando en bajos niveles de protección ${ }^{28}$. La práctica de hábitos de trabajo seguro en las prácticas hospitalarias puede ser aplicada sólo de manera parcial por los estudiantes. En los hospitales escuela son frecuentes los brotes de enfermedades y las infecciones zoonóticas ${ }^{6} \mathrm{y}$ las superficies rozadas por múltiples personas y pacientes pueden estar contaminadas por patógenos zoonóticos, aún en períodos no epidémicos $5,20,45$.

La ausencia de prácticas tan simples como la apropiada higiene de manos facilita la ocurrencia de brotes de enfermedades zoonóticas entre los estudiantes ${ }^{15}$. Sin embargo, esto puede ser rápidamente revertido con intervenciones educativas similares a las utilizadas en hospitales humanos ${ }^{39}$.

Al igual que en los graduados, las correlaciones entre las percepciones de riesgos en las distintas activi- dades tuvieron coeficientes relativamente bajos y no se detectaron asociaciones entre el uso de EPP y estas percepciones. Ambas variables tuvieron valores más altos en mujeres que hombres, dado que estos últimos, en general tienden a minimizar los riesgos para la salud y a considerarlos menos problemáticos que las mujeres ${ }^{3}$.

El 6\% de los estudiantes había sido diagnosticado con alguna zoonosis, siendo la dermatofitosis la afección más frecuente. Esta es una de las zoonosis más prevalente entre los estudiantes de veterinaria ${ }^{11}$ y está asociada a la práctica con pequeñas especies ${ }^{18}$. Por el contrario, la frecuencia de brucelosis en Argentina está relacionada al trabajo con grandes animales.

La prevalencia de anticuerpos anti-brucelosis varió entre 1 y $18 \%$ de los estudiantes muestreados en distintas regiones de Argentina, Brasil y Colombia 7, 27,41. Por su parte, la alta prevalencia de anticuerpos contra Toxoplasma gondii entre estudiantes fue demostrada en Brasil hace más de 40 años ${ }^{32}$, aunque la importancia relativa de su asociación con la exposición a gatos $\mathrm{u}$ otros factores de riesgo como los hábitos alimentarios en estudiantes, resulte aún controversial ${ }^{13,31}$. En Argentina y Brasil, la seroprevalencia en muestras no aleatorizadas fue estimada entre el 26 y el $29 \%{ }^{7,42}$.

Los ingresantes a la carrera de Veterinaria y los estudiantes ${ }^{8}$ pueden tener un conocimiento insuficiente sobre el impacto de las zoonosis transmitidas por la fauna silvestre, las mascotas y los animales productores de alimentos. La bioseguridad es un tema relativamente reciente entre los veterinarios ${ }^{23} \mathrm{y}$, hasta hace pocos años, en las carreras de grado no existía una enseñanza metódica de seguridad e higiene laboral y sólo algunas Facultades tenían programas y comisiones institucionales específicas sobre estos temas ${ }^{1}$.

Tal perspectiva ha ido cambiando con la actualización de las legislaciones vigentes y las exigencias de los procesos de acreditación de las carreras por parte de organismos nacionales y multilaterales. Los contenidos sobre bioseguridad deberían ser ofrecidos desde los primeros años de la carrera, para poder modificar comportamientos y reducir los riesgos, siendo necesario que los docentes aumenten la observación de prácticas seguras entre sus alumnos.

Agradecimientos. A la Agencia Nacional de Promoción Científica y Tecnológica, por la financiación del trabajo (PICT 14-1665).

Referencias. El artículo incluye más de 50 citas bibliográficas que no se insertaron por su larga extensión. Los interesados pueden solicitarlas a htarabla@fcv.unl. edu.ar 